\title{
Investigação Científica
}

\section{Conhecimento de cirurgiões-dentistas de Atenção Primária à Saúde acerca de urgências endodônticas}

Knowledge of Primary Health Care dentists about endodontic urgencies

Michele Marciano de Oliveira*

Francisco Montagner $^{* *}$

Victor Nascimento Fontanive ${ }^{* * *}$

\section{Resumo}

Objetivo: avaliar o conhecimento de um grupo de cirurgiões-dentistas que trabalham em Atenção Primária à Saúde acerca de diagnósticos e condutas a serem tomadas diante de urgências em endodontia. Métodos: 24 cirurgiões-dentistas responderam um questionário estruturado, adaptado para o estudo. O instrumento abordava características socioeconômicas e o conhecimento dos cirurgiões-dentistas sobre diagnósticos e condutas perante patologias pulpares e periapicais. Os dados foram submetidos a análises estatísticas descritivas (medidas de variabilidade e de tendência central) e de associação entre os desfechos e as variáveis independentes, pelo Teste $t$ Student e pelo teste de correlação Pearson, todos com $\alpha \leq 0,05$. Resultados: a quantidade de acertos dos participantes foi baixa nas perguntas selecionadas, as quais eram vinculadas aos conhecimentos de urgência em endodontia $(27,3 \%-68,2 \%)$. Essa baixa quantidade de acertos foi relacionada às variáveis sexo, idade e tempo de formado. Conclusão: o cirurgião-dentista deve estar preparado para lidar com urgências endodônticas desde a formação acadêmica. Considerando a baixa quantidade de acertos nas questões acerca de urgências endodônticas, atualizações constantes sobre o tema são necessárias, a fim de aprimorar o conhecimento dos profissionais e ampliar a resolutividade dos serviços.

Palavras-chave: Atenção Primária à Saúde. Conhecimento. Endodontia. Urgência.

Especialista em Saúde da Família e Comunidade pelo Grupo Hospitalar Conceição, Porto Alegre, RS, Brasil.

Doutor em Clínica Odontológica pela Universidade Estadual de Campinas. Professor Associado ao Departamento de Odontologia Conservadora, Área de Endodontia da Universidade Federal do Rio Grande do Sul.

* Mestre em Saúde Bucal Coletiva pela Universidade Federal do Rio Grande do Sul. Atua no Serviço de Saúde Comunitária do Grupo Hospitalar Conceição, Porto Alegre, RS, Brasil. 


\section{Introdução}

A inserção da saúde bucal e das práticas odontológicas na Estratégia de Saúde da Família (ESF) deu-se por meio da Portaria no 1.444 , de 28 de dezembro de 2000, sendo que aconteceu de maneira paralela e afastada do processo de organização dos demais serviços de saúde ${ }^{1}$. Entretanto, a consolidação das equipes de saúde bucal só ocorreu com a criação da Política Nacional de Saúde $\mathrm{Bucal}^{2}$. A saúde bucal passou a ser ofertada de forma integral e houve a inserção de procedimentos mais complexos na Atenção Básica, com a criação de uma rede de serviços de atenção em saúde bucal no Sistema Único de Saúde (SUS) ${ }^{3}$. Apesar de ter oportunizado melhorias e avanços, os serviços de assistência odontológica nem sempre são uma realidade para toda a população, e uma grande demanda ainda se revela desassistida. Diante desse panorama, ocorrem, frequentemente, as urgências ${ }^{4}$.

A dor que advém de urgências odontológicas pode ocorrer em razão de inúmeros problemas: endodônticos, periodontais ou, ainda, originários da articulação temporomandibular ${ }^{5}$. As principais causas que levam os pacientes à procura por tratamento de urgência são as doenças de origem pulpar ou perirradicular, sendo a dor o sintoma mais predominante ${ }^{6}$. A intervenção endodôntica local é imprescindível para alívio dos sintomas ${ }^{7}$. A dor impacta diretamente na qualidade de vida das pessoas, podendo afetar o sono, o lazer, o trabalho e os relacionamentos interpessoais ${ }^{8}$. Devido à sua alta prevalência, a dor é considerada um problema de saúde pública, gerando impacto negativo na qualidade de vida das pessoas, absenteísmos e custos econômicos relacionados aos serviços de saúde ou mesmo a medicamentos ${ }^{9}$.

Em endodontia, situações de urgência caracterizam-se por episódios de dor, associados ou não com tumefação, causada por inflamação ou infecção dos tecidos pulpares e/ou periapicais. Diante disso, a abordagem clínica varia de acordo com o estado pulpar ou periapical, com a intensidade e a duração da dor e com a presença de edema ${ }^{10,11}$. Os quadros clínicos mais frequentemente observados em urgências endodônticas são: pulpites (reversíveis e irreversíveis), abcesso periapical agudo, necrose pulpar, fraturas dentárias e periodontite (pericementite) apical ${ }^{6}$.

Visto que as urgências odontológicas constituem uma realidade no cotidiano do atendimento odontológico, é necessário que o cirurgião-dentista esteja capacitado para identificar as principais patologias pulpares e periapicais que ocorrem no serviço de Atenção Primária à Saúde (APS) ${ }^{12,13}$. Assim, o objetivo do estudo foi avaliar o conhecimento dos cirurgiões-dentistas que trabalham em Unidades de Saúde da APS em uma capital do sul do Brasil, acerca dos diagnósticos e das condutas terapêuticas a serem tomadas em relação a urgências em endodontia.

\section{Material e método}

O presente estudo apresenta delineamento transversal, do tipo descritivo exploratório, e abordagem quantitativa, tendo sido aprovado pelo Comitê de Ética em Pesquisa do Grupo Hospitalar Conceição, pelo parecer CAAE: 81307417.1.0000.5530.

Foram convidados a participar do estudo cirurgiões-dentistas vinculados a um serviço de APS de Porto Alegre, RS, Brasil. Os profissionais que aceitaram participar do estudo ratificaram seu interesse por meio da assinatura de um termo de consentimento livre e esclarecido.

A coleta de dados foi realizada por meio de um questionário elaborado para o estudo, contendo perguntas fechadas. Os questionários foram distribuídos em envelopes fechados, sendo recolhidos por meio de uma urna, visando garantir o sigilo, sem que o participante pudesse ser identificado.

O questionário abordava características socioeconômicas (perfil profissional, sexo, idade, tempo de formado, título de especialização, especialização em endodontia, tempo de trabalho na APS) e a respeito do conhecimento dos cirurgiões-dentistas sobre diagnósticos e condutas diante de patologias pulpares e periapicais. As questões sobre o conhecimento relacionado a patologias pulpares e periapicais foram adaptadas do estudo de Augusto $^{14}$ (2016).

Foram realizadas análises estatísticas descritivas (medidas de variabilidade e de tendência central) e de associação entre os desfechos e as 
variáveis independentes utilizando o programa SPSS 16.0, por meio do Teste $t$ Student e da análise de correlação Pearson, todos com $\alpha \leq 0,05$.

\section{Resultados}

Do universo da pesquisa, composto por 24 cirurgiões-dentistas de Unidades de Saúde de APS, 2 não responderam, totalizando uma taxa de resposta de 91,6\%. Quanto à caracterização do perfil profissional dos cirurgiões-dentistas respondentes, observou-se que a amostra foi composta por $7(31,8 \%)$ homens e $15(68,2 \%)$ mulheres, apresentando idades entre 29 e 67 anos, sendo a média de 45 anos. Com relação ao tempo de formado, os participantes do estudo apresentaram uma média de aproximadamente $22 \operatorname{anos}( \pm 10,71)$. Dos respondentes, $19(86,4 \%)$ possuíam título de especialista e $3(13,6 \%)$ eram clínicos gerais. Nenhum dos respondentes possuía título de especialista em endodontia. Em relação ao tempo de trabalho na APS, a média foi de 17 anos $( \pm 7,82)$. Foi questionado também se a formação desses profissionais forneceu base para a realização dos diagnósticos e tratamentos em endodontia, sendo que 21 profissionais $(95,5 \%)$ responderam que sim. A maioria dos dentistas, 20 (90,9\%), respondeu que se sente seguro para atender qualquer tipo de situação de urgência em endodontia.

Com relação aos conhecimentos dos cirurgiões-dentistas sobre urgências em endodontia, foram aplicadas quatro perguntas, as quais estão descritas na Tabela 1.

Tabela 1 - Questões abordando o conhecimento dos cirurgiões-dentistas acerca de urgências endodônticas e a porcentagem de acertos

\begin{tabular}{|c|c|}
\hline Pergunta & Acertos (\%) \\
\hline $\begin{array}{l}1 \text { - O cirurgião-dentista da Unidade Básica de Saúde da Família pode realizar os seguintes procedimentos endodônticos: } \\
\text { Resposta correta: Proteção pulpar direta ou indireta e pulpotomias. }\end{array}$ & 27,3 \\
\hline $\begin{array}{l}2 \text { - Qual o diagnóstico e a medicação intracanal de urgência que estão correlacionados corretamente? } \\
\text { Resposta correta: Todas as linhas estão corretas. } \\
\text { I - Pulpite aguda irreversível - Otosporin } ® \\
\text { II - Abscesso periapical agudo em fase inicial - Tricresol formalina } \\
\text { III - Abscesso periapical agudo em fase evoluída - Tricresol formalina }\end{array}$ & 45,5 \\
\hline $\begin{array}{l}3 \text { - Qual a conduta diante de um paciente adulto com dor intensa, pulsátil, contínua e espontânea no dente } 25 \text {, que piora ao } \\
\text { baixar a cabeça ou ao deitar e não alivia com analgésico? } \\
\text { Resposta correta: Anestesia, acesso, remoção do tecido pulpar, hemostasia, colocação de medicação intracanal (corticoste- } \\
\text { roide), selamento e prescrição de anti-inflamatório. }\end{array}$ & 68,2 \\
\hline $\begin{array}{l}4 \text { - Paciente adulto com dor intensa, pulsátil, espontânea e contínua e que piora com a mastigação. Testes de sensibilidade } \\
\text { negativos, percussão e palpação positivos. Presença de edema intraoral e flutuante, porém sem mobilidade dentária e alte- } \\
\text { rações no periápice. Qual diagnóstico e conduta diante do caso? } \\
\text { Resposta correta: Abcesso dentoalveolar agudo. Acesso endodôntico e medicação intracanal. Drenagem intraoral e trata- } \\
\text { mento endodôntico após a fase aguda. }\end{array}$ & 36,4 \\
\hline
\end{tabular}

Fonte: autores.

Na Tabela 2, uma associação estatística significativa foi encontrada entre a variável sexo e a quantidade de acertos. Não houve associação estatística significativa das variáveis especialização e segurança em relação aos acertos.
Tabela 2 - Análise referente às variáveis sexo, especialização, formação e segurança nos atendimentos de urgências

\begin{tabular}{|c|c|c|}
\hline Variável & Média de acertos (DP) & Valor de $\mathrm{p}^{*}$ \\
\hline Sexo & $\begin{array}{l}\text { Masculino } 1,1 \pm 1 \\
\text { Feminino } 2 \pm 0,8\end{array}$ & $0,03^{* *}$ \\
\hline Possui especialização & $\begin{array}{l}\text { Sim } 1,8 \pm 0,9 \\
\text { Não } 1,3 \pm 1,5\end{array}$ & 0,41 \\
\hline Formação suficiente & $\begin{array}{l}\text { Sim } 1,8 \pm 0,9 \\
\text { Não } 0 \text { DP - }\end{array}$ & 0,6 \\
\hline $\begin{array}{l}\text { Segurança para } \\
\text { atendimento }\end{array}$ & $\begin{array}{l}\operatorname{Sim} 1,7 \pm 0,9 \\
\text { Não } 2,5 \pm 0,7\end{array}$ & 0,27 \\
\hline
\end{tabular}


A Tabela 3 mostra associações estatísticas significativas entre idade e tempo de formado em relação ao número de acertos. Não houve associação estatística significativa entre a variável tempo de atuação na APS em relação à quantidade de acertos.

Tabela 3 - Correlação entre as variáveis idade, tempo de formado, tempo de atuação na APS e os acertos no questionário

\begin{tabular}{l|l|l|l}
\hline \multicolumn{1}{c|}{ Variável } & \multicolumn{1}{|c|}{$\begin{array}{c}\text { Média em anos } \\
(\mathrm{DP})\end{array}$} & $\begin{array}{c}\text { Valor } \\
\text { de } \mathrm{r}\end{array}$ & $\begin{array}{c}\text { Valor } \\
\text { de } \mathrm{p}\end{array}$ \\
\hline Idade & $45 \pm 11,3$ & $-0,48^{*}$ & $0,02^{* *}$ \\
Tempo de formado & $21,8 \pm 10,7$ & $-0,46^{*}$ & $0,03^{* *}$ \\
Tempo de atuação na APS & $17 \pm 7,8$ & $-0,27^{*}$ & 0,22 \\
\hline
\end{tabular}

Fonte: autores.

${ }^{*}$ Coeficiente de correlação Pearson.

** $\alpha 0,05$.

\section{Discussão}

O estudo mostrou que a quantidade de acertos dos participantes foi baixa nas perguntas selecionadas, as quais eram vinculadas aos conhecimentos de urgência em endodontia, variando entre $27,3 \%$ e $68,2 \%$. Esse resultado reforça os achados encontrados na literatura de que o conhecimento dos cirurgiões-dentistas acerca de diagnósticos e condutas em situações de urgências endodônticas é limitado ${ }^{14}$. Dessa forma, é necessária a atualização permanente, para que o cirurgião-dentista atenda os casos de urgência endodônticas de modo mais resolutivo, sendo esta necessidade identificada desde a graduação ${ }^{14,15}$.

Em nosso estudo, no que se refere à segurança para executar os atendimentos de urgências endodônticas, a grande maioria dos profissionais relataram que se sentem preparados. Quanto à formação acadêmica, a maioria dos respondentes referiu que a graduação forneceu base técnico-científica para realizar os diagnósticos e tratamentos em endodontia na APS. Alguns autores reforçam a necessidade de que os cirurgiões-dentistas estejam preparados para conduzir a resolução de determinados tipos de urgências odontológicas, pois as etapas clínicas influenciam diretamente no sucesso do tratamento ${ }^{14,16}$.

Com relação à variável especialidade, o presente estudo não identificou diferença estatísti- ca significativa entre os grupos. No entanto, alguns estudos mostram que o conhecimento está diretamente relacionado ao nível de atualização profissional $^{17-19}$. No presente estudo, nenhum cirurgião-dentista respondeu ser especialista em endodontia. Esse fato pode explicar a baixa quantidade de acertos nas questões referentes aos conhecimentos em endodontia, para tratamento das urgências endodônticas. Salienta-se que o cirurgião-dentista deve obter o conhecimento necessário para solucionar as patologias da polpa e do periápice ainda na graduação, visto que são conceitos básicos e relacionados à prática clínica do cirurgião-dentista ${ }^{16}$.

Dos participantes da pesquisa, a grande maioria era do sexo feminino, apresentando um maior percentual de acertos em comparação ao masculino. Esse resultado pode estar relacionado com a mudança no perfil dos profissionais, sendo que nos últimos anos a grande maioria dos egressos de graduação é do sexo feminino ${ }^{20,21}$. As mulheres também apresentam perfil de formação mais recente, com uma média de idade menor (42,2 anos) quando comparadas aos homens (51,2 anos). Alguns estudos mostram que profissionais com menos tempo de formados e mais atualizados obtêm melhor desempenho nas respostas ${ }^{17-19}$.

Corroborando essa análise, nosso estudo encontrou correlação estatística significativa entre as variáveis idade e tempo de formado e o número de acertos. Profissionais com menor idade e menor tempo de formação apresentaram maior percentual de acertos. Outros autores também demonstraram em seus estudos resultados semelhantes, relacionados a traumas dentários ${ }^{22,23}$. A mudança nos cursos de graduação e pós-graduação pode ter influência na quantidade de acertos $^{23}$. No presente estudo, não foi observado correlação entre a variável tempo de atuação na APS e o número de acertos obtidos.

É importante registrar que na literatura foram encontrados poucos estudos que analisaram o conhecimento dos cirurgiões-dentistas sobre as urgências endodônticas, tornando-se uma justificativa para esta realização. Cabe ressaltar, também, que o número de indivíduos participantes da pesquisa se apresenta como uma possível limitação do estudo, apesar de abranger o censo de cirurgiões- 
-dentistas do serviço pesquisado. Por fim, há que se considerar que o questionário adotado, tendo como referência uma região do Brasil, pode não conseguir contemplar totalmente as especificidades regionais das diferentes formações em endodontia.

\section{Conclusão}

O presente estudo evidenciou uma baixa quantidade de acertos acerca do conhecimento sobre urgências endodônticas em APS. Essa baixa quantidade de acertos foi relacionada às variáveis sexo, idade e tempo de formado. Dessa forma, fica evidente a necessidade de que o cirurgião-dentista esteja plenamente preparado para lidar com urgências endodônticas desde a formação acadêmica. Para isso, atualizações constantes sobre o tema são necessárias, a fim de aprimorar o conhecimento dos profissionais, ampliando a resolutividade dos serviços.

\section{Abstract}

Objective: To assess the knowledge of a group of dentists working in Primary Health Care about the diagnostics and behaviors to be adopted to treat endodontic emergencies. Methods: 24 dentists answered a structured questionnaire adapted to the study. The instrument addressed socioeconomic characteristics and the knowledge of dentists about diagnostics and behaviors concerning pulpal and periapical pathologies. The data were subjected to analyses of descriptive statistics (measures of variability and central tendency) and of association between the outcomes and the independent variables using Student's t-test and Pearson correlation test. Significance was set at $\alpha \leq 0.05$. Results: The number of correct answers was low in the questions selected, which were linked to the knowledge of endodontic emergencies (27.3 - 68.2\%). The low number of correct answers was related to the variables of sex, age, and time of academic training. Conclusion: Dentists should be prepared to deal with endodontic emergencies since their academic training. The low number of correct answers for questions about endodontic emergencies shows the need for constant updates on the subject, in order to improve the knowledge of the professionals and expand the resoluteness of services.

Keywords: Emergency; Endodontics; Knowledge; Primary Health Care.

\section{Referências}

1. Brasil. Ministério da Saúde. Secretaria de Atenção à Saúde. Departamento de Atenção Básica. Saúde Bucal. Brasília: Ministério da Saúde; 2008. [Acesso em: 26 jun. 2018]. Disponível em URL: http://bvsms.saude.gov.br/bvs/publicacoes/ saude_bucal.pdf.

2. Brasil. Ministério da Saúde. Secretaria de Atenção à Saúde. Departamento de Atenção Básica. Coordenação Nacional De Saúde Bucal. Diretrizes da Política Nacional de Saúde Bucal. Brasília: Ministério da Saúde; 2004. [Acesso em: 02 ago. 2018]. Disponível em URL: http://bvsms.saude.gov.br/bvs/ publicacoes/politica_nacional_brasil_sorridente.htm.

3. Brasil. Ministério da Saúde. Secretaria de Atenção à Saúde. Secretaria de Vigilância em Saúde. SB Brasil 2010: Pesquisa Nacional de Saúde Bucal: resultados principais. Brasília: Ministério da Saúde; 2012. [Acesso em: 15 jun. 2018]. Disponível em URL: http://bvsms.saude.gov.br/bvs/publicacoes/ pesquisa_nacional_saude_bucal.pdf.

4. Sanchez HF, Drumond MM. Atendimento de urgências em uma Faculdade de Odontologia de Minas Gerais: perfil do paciente e resolutividade. Revista Gaúcha de Odontologia 2011; 59(1):79-86.

5. Tortamano IP, Costa CG, Moraes LJ, Borsatti MA, Rocha RG, Tortamano N. As urgências odontológicas e o tratamento clínico e medicamentoso integrado. Jornal Brasileiro de Clínica Odontológica Integrada 2004; 8(43):78-85.

6. De-Paula KB, Silveira LS, Fagundes GX, Ferreira MB, Montagner F. Patient automedication and professional prescription pattern in an urgency service in Brazil. Braz Oral Res 2014; 28(1):1-6.

7. Siqueira JF, Rôças IN, Lopes HP. Emergências e urgências em endodontia. In: Lopes HP, Siqueira JF. Endodontia, biologia e técnica. 4. ed. Rio de Janeiro: Elsevier; 2015. p. 1272-1314.

8. Cavalheiro CH, Abegg C, Fontanive VN, Davoglio RS. Dental pain, use of dental services and oral health-related quality of life in southern Brazil. Braz Oral Res 2016; 30(1):39.

9. Miotto MHMB, Silotti JCB, Barcellos LA. Dor dentária como motivo de absenteísmo em uma população de trabalhadores. Ciência e Saúde coletiva 2012; 17(5):1357-63.

10. Wolcott J, Rossman LE, Hasselgren G. Controle das emergências endodônticas. In: Cohen S, Hargreaves K. Caminhos da polpa. 10. ed. Rio de Janeiro: Elsevier; 2011. p. 38-45.

11. Brasil. Ministério da Saúde. Secretaria de Atenção à Saúde. Departamento de Atenção Básica. Manual de especialidades em saúde bucal. Brasília: Ministério da Saúde; 2008. [Acesso em: 11 jun. 2018]. Disponível em URL: http://bvsms.saude.gov. br/bvs/publicacoes/manual_especialidades_saude_bucal.pdf.

12. Pinto EC, Barros VJA, Coelho MQ, Costa SM. Urgências odontológicas em uma Unidade de Saúde vinculada à Estratégia Saúde da Família de Montes Claros, Minas Gerais. Arquivos em Odontologia 2012; 48(3):166-74.

13. Albuquerque LAD, Archer CE, Souza RMS, Travassos RMC, Gomes SGF, Santos RA. Prevalência de doenças pulpares e periapicais na clínica de especialização em endodontia da FOP/UPE. Revista Cirurgia Traumatologia Buco-Maxilo-Facial Camaragibe 2011; 11(1):77-83.

14. Augusto SM. Conhecimento e práticas de cirurgiões-dentistas da estratégia saúde da família acerca das urgências endodônticas [Monografia de Graduação em Odontologia]. Campina Grande: Universidade Estadual da Paraíba; 2016.

15. Souza DP. Conhecimentos dos estudantes de odontologia sobre urgências endodônticas [Monografia de Graduação em Odontologia]. Campina Grande: Universidade Estadual da Paraíba; 2016. 
16. Bezerra PASAM, Oliveira MEM, Gomes TN, Barbosa JS, Medeiros EF. Principais patologias orofaciais recorrentes em serviços de urgências odontológicas: uma revisão de literatura. Revista de Psicologia 2015; 9(27):167-77.

17. Carvalho VAP, Borgatto AF, Lopes LC. Nível de conhecimento dos cirurgiões-dentistas de São José dos Campos sobre o uso de anti-inflamatórios não esteroides. Ciência \& Saúde Coletiva 2010; 15(1):1773-82.

18. Holanda GZ, Matos IV, Lima MCD, Silva PGP, Pereira PMH, Cavalcanti MSM, et al. Perfil da prescrição de colutórios, analgésicos, anti-inflamatórios e antibióticos por cirurgiões-dentistas na região metropolitana do Recife/PE. Rev. Cons Reg Odontol Pernambuco 2001; 4(1):49-57.

19. Cutrim MCFN, Reis FS, Valois EM, Lopes FF. Nível de conhecimento dos cirurgiões-dentistas sobre o câncer de boca na rede pública da cidade de São Luís-MA. Rev Odonto Ciência $2004 ; 19(45): 270-4$.

20. Celeste MM, Haddad AE, Araújo ME. Perfil atual e tendências do cirurgião-dentista brasileiro. Maringá: Dental Press; 2010. p. 47-55.

21 .Dib LL, Souza RS, Tortamano N. Avaliação do conhecimento sobre câncer bucal entre alunos de Odontologia, em diferentes unidades da Universidade Paulista. Rev Inst Ciênc Saúde 2005; 23(4):287-95.

22. Conhenca N, Forrest JL, Rotstein I. Knowledge of oral health professional of treatmente of avulsed teeth. Dent Traumatol 2006 ; 22(6):296-301.

23. França RI, Traebert J, Lacerda JT. Brazilian dentists knowledge regarding immediate treatment of traumatic dental injuries. Dent Traumatol 2007; 23(5):287-90.

Endereço para correspondência:

Michele Marciano de Oliveira

Rua Belo Horizonte, quadra 155, lote 08, Parque Amazônia

CEP 74843-100 - Goiânia, Goiás, Brasil

Telefone: +55 (51) 98578-0381

E-mail: michele-oliveira18@hotmail.com

Recebido: 09/06/19. Aceito: 09/09/19. 